archives-ouvertes

\title{
Effect of magnetophoresis and Brownian diffusion on mechanical processes in magnetic fluids: the role of a condensation phase transition
}

\author{
V. Bashtovoi, A. Reks, P. Kuzhir, A. Zubarev, O. Volkova, V. Moroz
}

\section{To cite this version:}

V. Bashtovoi, A. Reks, P. Kuzhir, A. Zubarev, O. Volkova, et al.. Effect of magnetophoresis and Brownian diffusion on mechanical processes in magnetic fluids: the role of a condensation phase transition. Journal of Magnetism and Magnetic Materials, Elsevier, 2020, 498, pp.166148. 10.1016/j.jmmm.2019.166148 . hal-02426099

\section{HAL Id: hal-02426099 \\ https://hal.archives-ouvertes.fr/hal-02426099}

Submitted on 1 Jan 2020

HAL is a multi-disciplinary open access archive for the deposit and dissemination of scientific research documents, whether they are published or not. The documents may come from teaching and research institutions in France or abroad, or from public or private research centers.
L'archive ouverte pluridisciplinaire HAL, est destinée au dépôt et à la diffusion de documents scientifiques de niveau recherche, publiés ou non, émanant des établissements d'enseignement et de recherche français ou étrangers, des laboratoires publics ou privés. 


\section{Effect of magnetophoresis and Brownian} diffusion on mechanical processes in magnetic fluids: the role of a condensation phase transition

\author{
V. Bashtovoi ${ }^{1}$, A. Reks ${ }^{1}$, P.Kuzhir ${ }^{2}$, A.Zubarev ${ }^{3}$, \\ O.Volkova $^{2}$, V.Moroz ${ }^{1}$ \\ ${ }^{1}$ Belarusian National Technical University, 65 \\ Nezavisimosti Ave., 220013, Minsk, Belarus \\ ${ }^{2}$ University Côte d'Azur, CNRS UMR 7010, Institute of \\ Physics of Nice, , Parc Valrose, Nice 06108, France \\ ${ }^{3}$ Ural Federal University, Lenin St.,51, 620002, \\ Ekaterinburg, Russia
}

\section{Abstract}

In this work, we study theoretically the effect of the mass transfer processes on the volume magnetic force and viscous friction of the magnetic fluid subjected to a magnetic field gradient and a shear flow between two rotating cylinders. The model is based on the diffusion equations and takes into account a condensation phase transition in the magnetic fluid. The results of experimental and theoretical studies of the diffusion processes in a thin layer of the magnetic fluid are also presented.

Keywords: magnetic fluid; magnetophoresis; diffusion; phase transition; magnetic force; viscosity

\section{Introduction}

Magnetophoresis and Brownian diffusion are natural processes taking place in magnetic fluid, as a suspension of magnetic particles, in the presence of inhomogeneous magnetic fields $[1,2]$.

As a result of these processes the concentration of magnetic particles in a magnetic fluid does not remain homogeneous and concentration gradients appear.

On the other hand, the physical properties of the magnetic fluid depend on the concentration of particles $\Phi$ [1-3], such that the following phenomena can appear:

1) the dependence of the magnetic fluid magnetization $M$ on $\Phi$ leads to a change of the magnetic volume force $F_{m}$ acting on the fluid [4];

2) the dependence of the fluid viscosity $\eta$ on $\Phi$ leads to a change of the viscous stresses in the fluid and the forces of viscous friction $F_{\tau}$ on the solid boundaries [5];

Furthermore, the nanoparticle concentration in some regions of the magnetic fluid can reach significant values, such as "gas- liquid" or "order-disorder" phase transitions can occur, changing nanoparticle spatial organization within concentrated phases. These phase transitions have been extensively studied in external homogeneous magnetic fields [2, 6-11], and are expected to affect the mass transfer properties under non-uniform magnetic fields. In this context, we are aimed in the present paper to establish the effect of the condensation phase transition under non-uniform field on (a) volume magnetic force and viscous friction force of the magnetic fluid subjected to shear flow between two rotating cylinders; (b) diffusion process in a thin layer of the magnetic fluid. The objective (a) has a practical significance for the proper design of magnetic fluid seals.

\section{Governing equations and phase transition conditions}

Mass transfer in a magnetic fluid associated with magnetophoresis and Brownian diffusion is governed by the diffusion equation

$\rho \frac{\partial \Phi}{\partial t}+\operatorname{divi}=0$

with the mass flux density $i$ given by the following equation, valid in a dilute limit $[2,4]$ :

$\boldsymbol{i}=-\rho D \nabla \Phi+\rho b \mu_{0} m \Phi L(\xi) \nabla H$,

where $t$ is the time, s; $\rho$ - density of particles, $\mathrm{kg} / \mathrm{m}^{3} ; D$ - diffusion coefficient, $\mathrm{m}^{2} / \mathrm{s}$, which is connected with the hydrodynamic mobility of particles $b$ by the Einstein's relation $D=k T b$; $\mu_{0} \approx 1.26 \cdot 10^{-6}, \mathrm{H} / \mathrm{m}$ - magnetic permeability of vacuum; $m$ - magnetic moment of individual particle, $\mathrm{J} / \mathrm{T} ; k \approx 1.38 \cdot 10^{-23}, \mathrm{~J} / \mathrm{K}$ - Boltzmann constant; $T$ - temperature, $\mathrm{K} ; L(\xi)=\operatorname{cth}(\xi)-1 / \xi$ - Langevin function, $\xi=\mu_{0} m H / k T$ - Langevin parameter.

The development of diffusion theory for concentrated magnetic fluids was carried out in $[12,13]$, however, as the first step, we will use more simple equations (1), (2) with constant diffusion coefficient.

At stationary state, the mass flux density is equal to zero in the whole volume of the fluid bounded by impermeable boundaries: $\boldsymbol{i}=0$.

These equations suppose that magnetic particles in the fluid behave like gas molecules. but as it was mentioned above in strong magnetic field there is a phase transition in magnetic fluid when magnetic particles form a condensed phase. This transition will be assumed as a natural limit of validity of the theory of Brownian diffusion [14].

So we will assume that magnetic particles in magnetic fluid can be in two states: "gas phase" 
where distribution of their concentration $\Phi_{\mathrm{g}}$ is governed by the diffusion equations (1), (2) valid under dilute limit approximation, and "condensed phase" where their concentration has a constant value $\Phi_{c}$. Conditions of the phase transition are defined by the dipolar coupling parameter $\lambda=\left(\mu_{0} m^{2}\right) /\left(4 \pi d^{3} k T\right)$, where $d$ is the particle diameter.

The dependence of the magnetic fluid magnetization $M$ on $\Phi$ will be described by $M=M_{s p} \Phi L(\xi)$ in the gas phase and by the following equation taking into account for pair dipolar interactions between particles in the condensed phase [7]: $M=M_{s p} \Phi L(\xi)(1+8 \Phi \mathrm{d} L / \mathrm{d} \xi)$, where $M_{s p}$ is the magnetization of one magnetic particle.

The Krieger-Dougherty approximation [15] will be used for the magnetic fluid viscosity $\eta=\eta(\Phi=0)(1-1.35 \Phi)^{-1.85}$.

Let us now consider two specific problems related to the effect of the condensation phase transition of the magnetic fluid behavior under non-uniform magnetic fields.

\section{Couette flow between concentric cylinders. Theory.}

Magnetic fluid $M F$ with constant temperature is held in a gap between coaxial cylinders of radii $R_{1}$ and $R_{2}$ rotating with angular velocities $\omega_{1}$ and $\omega_{2}$ by a locally inhomogeneous magnetic field created by an annular magnet pole $M P$. The problem geometry is presented in Fig. 1 and represents the model of a magnetic fluid seal.

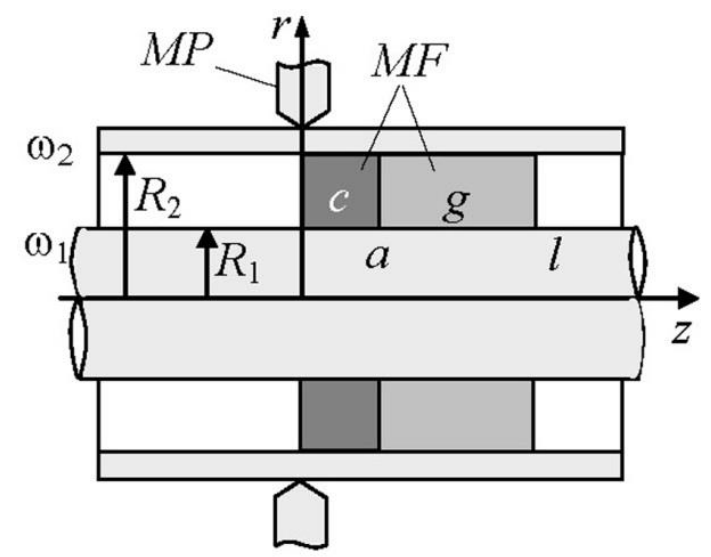

Fig. 1. Problem geometry of a magnetic fluid flow between rotating cylinders.

The assumption of the isothermal flow can be justified by evaluating the temperature difference $\Delta T$ across the gap between two rotating cylinders. Balancing the heat power generated by viscous dissipation and the heat flux evacuated through the interface between the magnetic fluid and the external cylinder, we get $\Delta T \sim \eta \omega^{2} R_{1}^{2} / \lambda_{e}$, where $\omega=\left|\omega_{1}-\omega_{2}\right|, \eta$ is the $M F$ viscosity, and $\lambda_{e}-$ its thermal conductivity. For the following realistic parameters of the $M F$ seal, $\eta \sim 10^{-2} \mathrm{~Pa} . \mathrm{s}, \omega \sim 2 \pi .50$ $\mathrm{rad} / \mathrm{s}(\sim 3000 \mathrm{rpm}), R_{1} \sim 10^{-2} \mathrm{~m}, \lambda_{e} \sim 0.1 \mathrm{~W} /(\mathrm{m} . \mathrm{K})$, we evaluate $\Delta T \sim 1 \mathrm{~K}$. Such a small temperature difference (with respect to a typical temperature $T \sim 300 \mathrm{~K}$ ) can be safely neglected and the flow can be considered as isothermal.

Furthermore, all the effects related to magnetization relaxation under shear flow are neglected because the typical relaxation time $\tau_{R} \sim 10^{-6}-10^{-5} \mathrm{~s}$ for the nanoparticle size $d=10-12$ $\mathrm{nm}$ [1] is much shorter than the hydrodynamic timescale $\tau_{H} \sim\left(R_{2}-R_{1}\right) /\left(\omega R_{1}\right) \sim 10^{-4}-10^{-3} \mathrm{~s}$.

The equilibrium location of the magnetic fluid volume that is not symmetrical with respect to the magnetic pole can depend on a pressure drop at its lateral boundaries, such as, for example, in a magnetic-fluid seal.

Due to magnetophoresis, the concentration of magnetic particles under the magnet pole $M P$ could substantially increase and a condensed phase can occur. So, the magnetic fluid volume $M F$ at $0<z<l$ (where $l$ is the length of the fluid layer) is considered to consist of two parts: gas phase, indicated by the character " $g$ " and condensed phase indicated by the character " $c$ ". The axial coordinate of the interface between these phases is denoted by $a$. Neglecting the surface tension and magnetic field variation across the gap between two cylinders, the lateral boundaries of the volume are assumed plane. The stationary solution of the problem is presented below.

Further in this section, the magnetic field is approximated using experimental data [5] in magnetic fluid seal as, $H^{\prime}=H / H^{*}=1 /\left[1+3(z / l)^{2}\right]$ where $H^{*}=1.6 \cdot 10^{6}$

$\mathrm{A} / \mathrm{m}$ is a characteristic value of the magnetic field intensity taken as a maximal value under the pole at $z=0, l=10 \mathrm{~mm}$.

Neglecting the magnetic field variation across the gap (under the thin gap limit $\left.\left(R_{2}-R_{1} / R_{1}<<1\right)\right)$ and under the aforementioned assumptions of the isothermal flow and equilibrium magnetization, one can show that the flow velocity has the only non-zero azimuthal component depending on the radial coordinate $r$, $v_{\varphi}=v(r)$, while another physical quantities depend only on the axial component $z$ : $\Phi=\Phi(z)$, $M=M[\Phi(z)], \eta=\eta[\Phi(z)]$.

For this problem, the fluid velocity distribution $v(r)$ are given by known solutions of the Navier-Stokes equations [16]: 
$v=\frac{\omega_{2} R_{2}^{2}-\omega_{1} R_{1}^{2}}{R_{2}^{2}-R_{1}^{2}} r+\frac{\left(\omega_{1}-\omega_{2}\right) R_{1}^{2} R_{2}^{2}}{R_{2}^{2}-R_{1}^{2}} \frac{1}{r}$.

The magnetic force acting by volume of the fluid, $F_{m}$, and the moment of viscous forces $N$ acting on each of the cylinders, are given by the following expressions:

$$
F_{m}=\mu_{0} M_{s p} \pi\left(R_{2}^{2}-R_{1}^{2}\right) \int_{l}^{a} \Phi(z) L(\xi) \frac{\mathrm{d} H}{\mathrm{~d} z} \mathrm{~d} z
$$

in the gas phase, and

$F_{m}=\mu_{0} M_{s p} \pi\left(R_{2}^{2}-R_{1}^{2}\right) \int_{a}^{0} \Phi(z) L(\xi)\left[1+8 \Phi(z) \frac{\mathrm{d} L}{\mathrm{~d} \xi}\right] \frac{\mathrm{d} H}{\mathrm{~d} z} \mathrm{~d} z$

in the condensed phase,

$N=2 \pi R_{1}^{2}\left[\frac{\mathrm{d} v}{\mathrm{~d} r}-\frac{v}{r}\right]_{r=R_{1}} \int_{0}^{l} \eta[\Phi(z)] \mathrm{d} z$ in the whole volume of $M F$ including the gas and the condensed phases.

The dimensionless variables as follows will be hereinafter used : $z^{\prime}=z / l, H^{\prime}=H / H^{*}$, $s=a / l, \quad \eta^{\prime}=\eta\left[\Phi\left(z^{\prime}\right)\right] / \eta\left(\Phi_{0}\right)$, where: $\Phi_{0}$ is an initial uniform concentration of particles.

The effect of the redistribution of the concentration of particles in a magnetic fluid will also be characterized by dimensionless coefficients representing the ratio of the physical quantity under consideration to its value at the uniform distribution of particles:

1) the relative magnetic force acting by magnetic fluid volume, $k_{m}=F_{m} / F_{m}\left(\Phi_{0}\right)$,

2) the relative moment of viscous forces,

$k_{\tau}=N / N\left(\Phi_{0}\right)=\int_{0}^{1} \eta^{\prime}\left(z^{\prime}\right) \mathrm{d} z^{\prime}$,

In relative values, all the coefficients associated with the radii of the cylinders and the angular velocities of their rotation are worked out and these values are independent of them.

The distribution of particle concentration $\Phi$ in the gas phase is defined by the expression (2) under dilute limit approximation, which taking into account the impermeability of boundaries, gives $i_{\mathrm{z}}=0$ at stationary state, or

$$
\frac{\mathrm{d} \Phi}{\mathrm{d} z^{\prime}}=U \Phi L\left(U H^{\prime}\right) \frac{\mathrm{d} H^{\prime}}{\mathrm{d} z^{\prime}} .
$$

This equation has a general solution

$\Phi_{g}=B \frac{s h\left[U H^{\prime}\left(z^{\prime}\right)\right]}{H^{\prime}\left(z^{\prime}\right)}$.

The main dimensionless parameter characterizing the problem is magnetic parameter $U=\mu_{0} m H^{*} / k T$ - a ratio of the particle magnetic energy to its thermal energy.
The integration constant $B$ and the axial coordinate of the interface between phases $s$ are defined from the condition of constancy of the average concentration $\Phi_{0}$,

$\Phi_{c} s+\int_{s}^{1} \Phi_{g} d z^{\prime}=\Phi_{0}$

To close the problem, one needs to specify the values of the concentration on the interface in the gas phase $\Phi_{g^{*}}$ and in the condensed phase $\Phi_{c}$. This specification can be done by using the analytical expressions given in [5] or by using the phase equilibrium diagram given below in Fig. 2. We will use the second way and define these concentrations at $\lambda=1.5$ we get $\Phi_{g^{*}}=0.489$, and $\Phi_{\mathrm{c}}=0.552$.

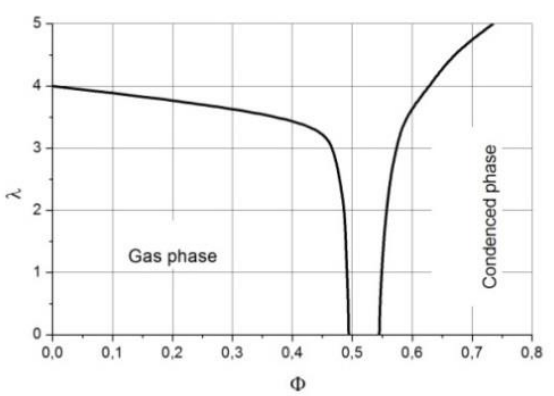

Fig. 2. Diagram of phase equilibrium in a magnetic fluid at saturation magnetization [5].

The results of calculations performed by the formulas (3)-(7) are presented in Figs. 3-5.

As one can see from Fig. 3, an increase of the particles concentration in the direction of regions with larger magnetic field intensity occurs monotonically up to a certain value of the magnetic parameter $U$ without formation of a condensed phase. When this value is exceeded (at

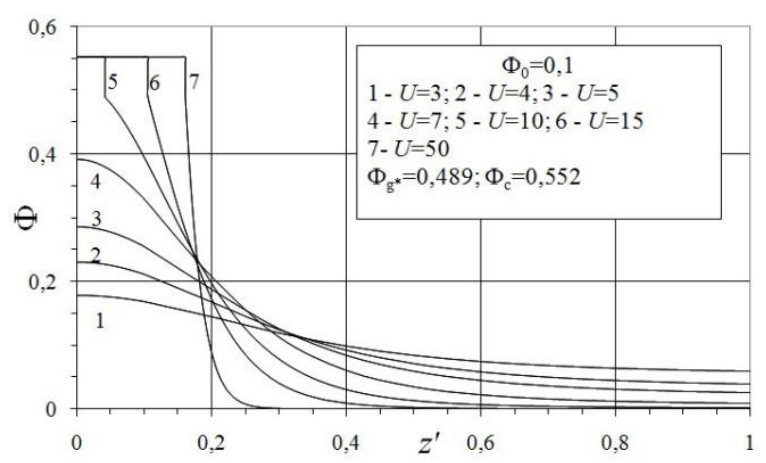

Fig. 3. Distribution of particle concentration $\Phi$ in magnetic fluid depending on magnetic parameter $U$ at initial concentration $\Phi_{0}=0.1$ and $H^{\prime}=1 /\left(1+3 z^{2}\right)$.

approximately $U>9$ for the conditions of the above calculations), the phase transition interface forms at $z=0$ were magnetic field intensity has a maximum. 
The coordinate of the phase transition interface $s$ shifts to the larger values of $z$ coordinate and the width of condensed phase region increases with growth of $U$.

Fig. 4 demonstrates that the reconcentration of particles has the largest effect on the value of viscous forces $\left(k_{\tau}\right)$, increasing them more than twice. The magnetic force increases at small values of $U$ and decreases at large $U$. The latter effect is due to the fact that this force is also determined by the value of the magnetic field gradient, and for the large $U$ a significant number of particles are concentrated near $z=0$, where this gradient is small.

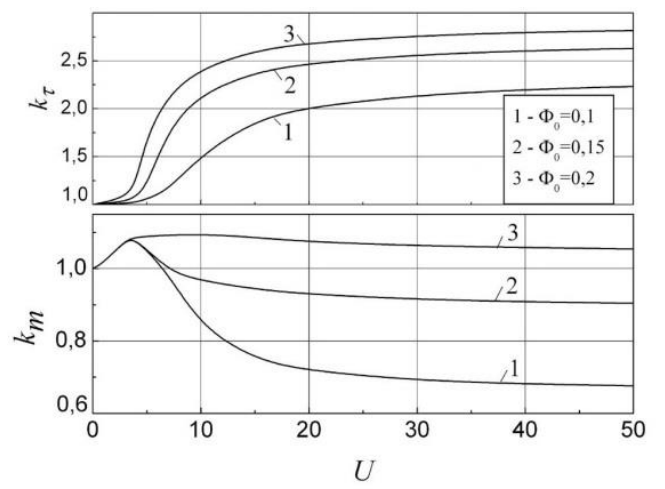

Fig. 4. Effect of the redistribution of particle concentration on the relative magnetic volume force $k_{m}=F_{m}(\Phi) / F_{m}\left(\Phi_{0}\right)$, and on the relative force of viscous friction $k_{\tau}=F_{\tau}(\Phi) / F_{\tau}\left(\Phi_{0}\right)$ at $\Phi_{g^{*}}=0.489$, and $\Phi_{\mathrm{c}}=0.552$.

\section{Magnetophoresis in axisymmetric plane layer.}

\section{a) experiment}

In order to establish some characteristics of the mass transfer processes described in Secs. 2, 3 , an experiment on diffusion and magnetophoresis was done in a thin axisymmetric

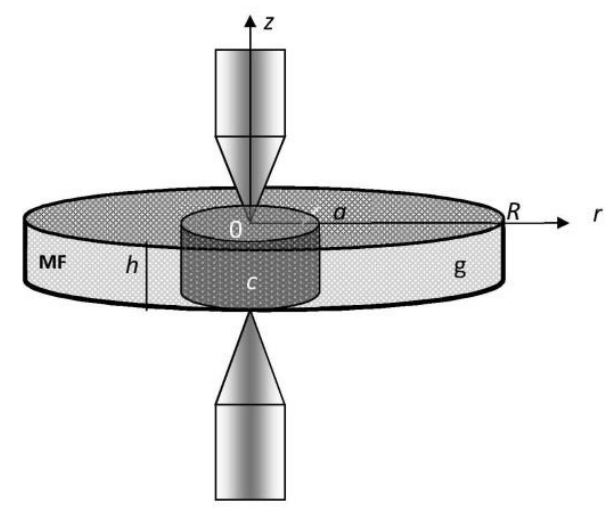

Fig. 5. Problem geometry of the plane axisymmetric magnetic fluid layer. plane magnetic fluid layer placed in axisymmetric magnetic field created by peaked poles of a permanent magnet as presented in Fig. 5.

Magnetic fluid layer has a thickness of $h=$ $0.06 \mathrm{~mm}$, and radius of $R=5.6 \mathrm{~mm}$.

In this case the distribution of magnetic field intensity is $H^{\prime}=H / H^{*}=1 /\left(1+2.5 r^{\prime 2}\right)$, where $H^{*}=450 \mathrm{KA} / \mathrm{m}$ is a maximal magnetic field intensity in the center of the layer; $r^{\prime}=r / R$.

Magnetic fluid based on kerosene and magnetite with saturation magnetization of $5 \mathrm{\kappa A} / \mathrm{m}$, density of $886 \mathrm{~kg} / \mathrm{m}^{3}$, initial volume fraction of particles, equal to 0.016 , was used. In this case $U=32, \lambda=1.5$.

Small concentration of particles and small thickness of the layer provide conditions for visualization of the process. Results of the experiment are presented in Figs. 6, 7.

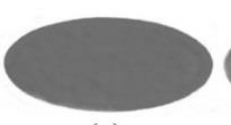

(a)

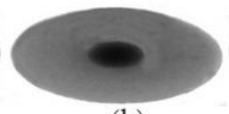

(b)

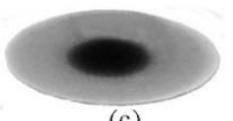

(c)
Fig. 6. Photos of the magnetic fluid layer at different moments of time: (a) $-0 \mathrm{~min}$, (b) $-120 \mathrm{~min},(\mathrm{c})-240 \mathrm{~min}$.

The increase of the black level $C$ with time in the central part of the layer in Fig. 6 characterizes the increase of the particle concentration.

The process of the concentration redistribution was recorded with time and the black level was assessed at each radial position $r$ at different elapsed times using the Adobe Photoshop software and the results are presented in Fig.7.

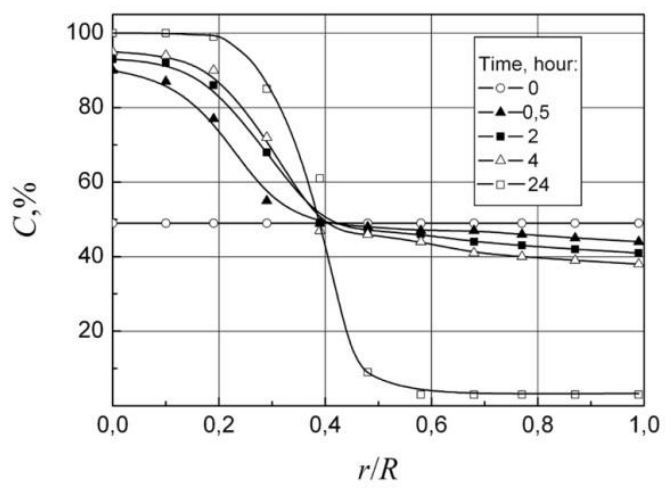

Fig. 7. The distribution of the black level $C$ of images along the radius of the magnetic fluid layer at different moments of time.

First of all, the experiment demonstrates a significant redistribution of the concentration of particles in a magnetic fluid in a highly inhomogeneous magnetic field of high intensity. 
As calibration measurements showed, at a particle concentration equal to or higher than 0.2 , the layer of magnetic fluid becomes opaque and has $C=100 \%$. Thus, in the experimental curves, the region in the central part of the layer $r / R<0.2$ is a region of concentrated fluid with a particle concentration larger than 0.2 , which is more than 10 times the initial concentration.

A more detailed experimental gradation of the particle concentration in the region $r / R<0.2$ becomes impossible due to fluid opaqueness and the determination of its properties requires further special studies.

\section{b) theory}

The theoretical description of this onedimensional problem under stationary condition (at long elapsed time after putting the sample under magnetic field) is completely analogous to the previous problem and has the same solution of the diffusion equation

$$
\Phi_{g}=B \frac{s h\left[U H^{\prime}\left(r^{\prime}\right)\right]}{H^{\prime}\left(r^{\prime}\right)},
$$

but the condition for the constancy of the average particle concentration will have a form different from (7), namely

$$
\Phi_{c} s^{2}+2 \int_{s}^{1} \Phi_{g} r^{\prime} d r^{\prime}=\Phi_{0}
$$

This, in particular, leads to the fact that for large values of $U$, when the main fraction of particles is concentrated in the "condensed" phase and the first term in (8) becomes much larger than the second one, the coordinate $s$ of the phase transition is determined by the relation $s \approx \sqrt{\Phi_{0} / \Phi_{c}}$ instead of the linear dependence $s \approx \Phi_{0} / \Phi_{c}$, as in the previous problem described in Sec. 3.

The distribution of the particle concentration $\Phi$ at initial concentration $\Phi_{0}=0.016$ in this case is presented in Fig.8 for different values of $U$.

The experimental curve for large time $t=24 \mathrm{~h}$ in Fig. 7 and the theoretical curve 3 for stationary state in Fig. 8 demonstrate adequate qualitative description of experimental data by the theory.

Over time, a region of high particle concentration, in accordance with both experiment and theory, is formed within $0<r / R<0.2$ where the particle concentration is larger than 0,2 and the "condensed" phase is formed in accordance with theory.

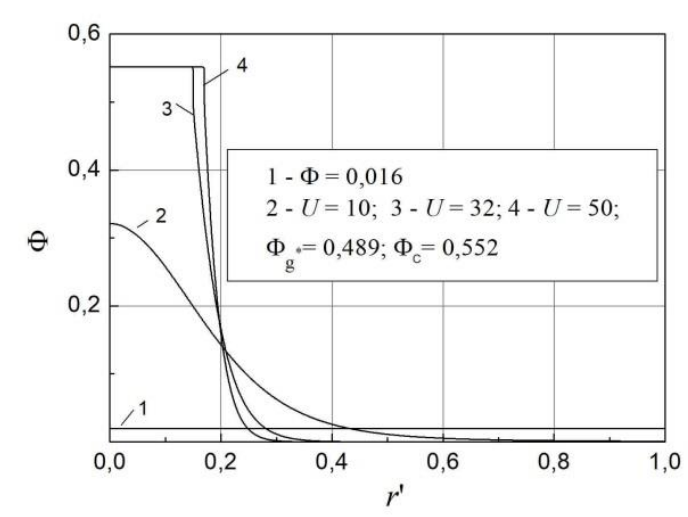

Fig. 8. Distribution of particle concentration $\Phi$ in axisymmetric plane magnetic fluid layer depending on magnetic parameter $U$ at initial concentration $\Phi_{0}=0.016$ and $H^{\prime}=1 /\left(1+2.5 r^{\prime 2}\right)$.

\section{Conclusions}

It is theoretically shown that the combination of the redistribution of the magnetic particle concentration in magnetic fluid (due to magnetophoresis and Brownian diffusion) and the condensation phase transition can essentially increase the viscous force under shear flow, while the effect on the volume magnetic force depends on the initial particle concentration and the value of the parameter $U$. Experiments and modelling of the mass transfer in an axisymmetric plane layer reveal a significant redistribution of the concentration of particles in the magnetic fluid in a highly inhomogeneous magnetic field and importance of the condensation phase transition on the concentration distribution.

\section{Acknowledgements}

One of the authors (PK) acknowledges financial support from the French ANR Project Future Investments UCA JEDI, No. ANR-15-IDEX-01 (projects ImmunoMag and MagFilter).

\section{References}

[1] R.E.Rosensweig, Ferrohydrodynamics, Cambridge University Press, New York, 1985, p. 344.

[2] E.Blums,A. Cebers, M.M.Maiorov. Magnetic Fluids, Water de Gruyter, Berlin, 1997, p. 430.

[3] V.G. Bashtovoy, B.M. Berkovsky, A.N. Vislovich, An Introduction to Thermomechanics of Magnetic Fluids, Hemisphere Publ. Corp., Washington, 1988, p. 190.

[4] V.G.Bashtovoi, V.K.Polevikov, A.E.Suprun, A.V.Stroots, S.A.Beresnev, Influence of Brownian diffusion on statics of magnetic fluid, Magnetohydrodynamics 43 (1) (2007) 17-26. [5] V.G. Bashtovoi, P.P. Kuzhir, A.Y. Zubarev, V.S. Moroz, On the mechanics of magnetic fluids with field-induced phase transition: application to Couette flow, Magnetohydrodynamics 54 (3) (2018) 181-196.

[6] A. O. Tsebers, Thermodynamic stability of magnetofluid, Magnetohydrodynamics 18 (2) (1982) 137-142.

[7] Y. A.Buyevich, A. O. Ivanov, Equilibrium properties of ferrocolloids. Physica A 190 (3-4) (1992) 276-294. 
[8] J. Liu, E. M. Lawrence, A.Wu, M. L. Ivey, G. A. Flores,

K. Javier, J. Bibette, and J. Richard, Field-Induced Structures in Ferrofluid Emulsions, Phys. Rev. Lett. 74 (14) (1995)

2828-2831.

[9] A.Y.Zubarev, L.Y.Iskakova, Theory of structural transformations in ferrofluids: chains and gas-liquid phase transitions, Phys. Rev. E 65 (2002) 061406.

[10] A. P. Hynninen and M. Dijkstra, Phase Diagram of Dipolar Hard and Soft Spheres: Manipulation of Colloidal Crystal Structures by an External Field, Phys. Rev. Lett. 94 (13) (2005) 138303

[11] E. Dubois, R. Perzynski, F. Bou'e, and V. Cabuil, Liquid-Gas Transitions in Charged Colloidal Dispersions: Small-Angle Neutron Scattering Coupled with Phase Diagrams of Magnetic Fluids, Langmuir 16 (13) (2000) 5617-5625

[12]. A. F. Pshenichnikov, E.A. Elfimova, A. O. Ivanov, Magnetophoresis, sedimentation, and diffusion of particles in concentrated magnetic fluids, J. Chem. Phys. 134, (2011) 184508.

[13]. A.S.Ivanov, A.F.Pshenichnikov, Magnetophoresis and diffusion of colloidal particles in a thin layer of magnetic fluids, J. of Magnetism and Magnetic Materials 322 (17) (2010) 25-75-2580.

[14] C.Magnet, P.Kuzhir, G.Bossis, A.Meunier, S.Nave, A.Zubarev, C.Lomenech, V.Bashtovoi, Behavior of nanoparticle clouds around a magnetized micro-sphere under magnetic and flow fields, Phys. Rev. E 89 (2014) 032310.

[15] I. M. Krieger, T. J. Dougherty, A mechanism for nonNewtonian flow in suspensions of rigid spheres, Trans. Soc. Rheol. 3 (1) (1959) 137-152.

[16] L. D. Landau and E. M. Lifshitz, A Course in

Theoretical Physics-Fluid Mechanics,Vol. 6, Pergamon, NewYork, 1987. 